\title{
Identification of KIF15 as a potential therapeutic target and prognostic factor for glioma
}

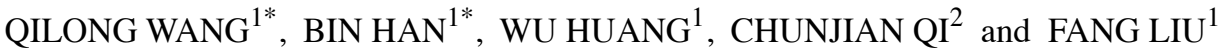 \\ Departments of ${ }^{1}$ Neurosurgery and ${ }^{2}$ Central Lab, Nanjing Medical University \\ Affiliated Changzhou No. 2 People's Hospital, Changzhou, Jiangsu 213003, P.R. China
}

Received April 11, 2019; Accepted November 18, 2019

DOI: $10.3892 /$ or.2020.7510

\begin{abstract}
Glioma is the most commonly diagnosed primary intracranial malignant tumor with rapid growth, easy recurrence and thus poor prognosis. In the present study, the role of kinesin-12 (KIF15) in glioma was revealed. Immunohistochemical staining and western blot analysis were used to detect the protein expression. An MTT assay was performed to evaluate cell proliferation. Flow cytometric analysis was utilized to assess cell apoptosis and the cell cycle. A mouse xenograft model was constructed for in vivo study. The results indicated that KIF15 was significantly upregulated in glioma tumor tissues and positively correlated with pathological staging, recurrence risk and poor prognosis. Silencing of KIF15 could inhibit cell proliferation and stemness of glioma cells, arrest cells in the G2 phase and induce cell apoptosis. The in vivo study verified the inhibitory effect of KIF15 knockdown on tumor growth. The mechanism study demonstrated the regulation of apoptosis- and cycle-related proteins in the KIF15 KD-induced inhibition of glioma. KIF15 was revealed to function as a tumor promoter in the development and progression of glioma. KIF15 also served as a prognostic indicator for glioma and may be a therapeutic target for glioma therapy.
\end{abstract}

\section{Introduction}

Glioma originates from glial cells of the brain and is one of the most commonly diagnosed intracranial tumors, accounting for $\sim 40$ to $50 \%$ of central nervous system tumors $(1,2)$. Malignant gliomas (stages III or IV, according to the World Health Organization) account for $\sim 77.5 \%$ of all gliomas (3). Malignant

Correspondence to: Dr Fang Liu, Department of Neurosurgery, Nanjing Medical University Affiliated Changzhou No. 2 People's Hospital, 68 Gehu Road, Changzhou, Jiangsu 213003, P.R. China

E-mail: czdoctorliu@hotmail.com

${ }^{*}$ Contributed equally

Key words: glioma, KIF15, knockdown, tumor promoter gliomas, especially glioblastoma (GBM), have a high incidence rate, high recurrence rate post-operation, high mortality rate and low cure rate (3). The most detrimental biological feature of malignant gliomas is the infiltrative growth of cancer cells, and the tumor tissues that cannot frequently be completely removed through surgery (4). Although advancements have been made in the pathogenesis, diagnosis and treatment of glioma recently, the prognosis of glioma, especially glioblastoma, is still not satisfying; with a 2 year survival rate of only $9 \%(5,6)$. At present, the clinical diagnosis of glioma depends on the results of histopathological examination, however the targets are usually difficult to identify (7). Therefore, it is of great importance to seek targets for early diagnosis, to assess therapeutic effects and for prognostic prediction of glioma. Although some markers have been recognized for glioma, such as human cartilage glycoprotein 39 (YKL-40), EphA2, CD133 and matrix metalloproteinase (MMP)-9, identification of more accurate and specific targets are still required for patients with glioma $(8,9)$.

The kinesin protein superfamily (KIF) is a type of molecular motor, which plays an important role in mitosis, organelle transport, meiosis, signal transduction, protein transport and several other cellular functions (10). Following the first naming of kinesin by Hirokawa and Tanaka (11), dozens of kinesin proteins have been identified up to date. Two types, namely conventional kinesin and unconventional kinesin, can be determined according to the different functions of kinesin proteins. The former has intracellular transport activity, such as KIF1A and KIF5B, whereas the latter does not exhibit ATP enzyme activity and cannot play a role in intracellular material transport $(12,13)$. Previous studies have revealed that kinesin proteins are associated with neurodegenerative diseases such as Alzheimer's disease (14) and Huntington's disease (15), diabetes (16) and nephropathy (17). Moreover, aberrant kinesin expression could induce the alteration of the distribution of intracellular genetic material, which could progressively lead to tumorigenesis, through chromosomal over-agglutination, spindle formation abnormalities, cell division defects or aneuploidy, and mitotic arrest (18). Accumulating evidence has revealed that kinesin proteins are widely involved in the occurrence and development of a variety of tumors, and their expression levels are also directly associated with the occurrence and development of many tumors $(19,20)$. 
Kinesin-12, also known as KIF15, is a microtubule-dependent motor protein and participates in mitosis and neuronal development (21). A previous study reported that KIF15 was a critical regulator in the promotion of pancreatic cancer proliferation through MEK/ERK signaling pathway (22). Milic et al revealed that KIF15-IN-1, an inhibitor of KIF15, could suppress cancer cell growth (23). However, the role of KIF15 in promoting the development of glioma and its potential as a therapeutic target need to be determined. Therefore, the present study demonstrated for the first time that the expression of KIF15 was upregulated in glioma tissues and was positively associated with the pathological staging, recurrence risk and poor prognosis. Moreover, the knockdown (KD) of KIF15 could significantly inhibit the development and stemness of glioma, indicating that KIF15 may be a potential therapeutic target for the treatment of glioma.

\section{Materials and methods}

Materials. The following materials were used: U87 MG cell line (glioblastoma of unknown origin; Shanghai Fuheng Biological Technology), U251 (BeNa Technology). The cells were both authenticated by short tandem repeat profiling. BR-V-108 (Shanghai Bioscienceres) was used as a plasmid vector from TOP10 E. coli competent cells (cat. no. CB104-03; Tiangen Biotech Co.,Ltd.). D-Hanks and trypsin were obtained from Shanghai Chemical Reagent Company) and the KIF15 antibody was purchased from Fine Test (cat. no. FNab04551).

Six-week-old male BALB/c nude mice were purchased from Shanghai Jake BIO Technology Co., Ltd. and divided into two groups randomly with 6 mice in each group. All mice were housed under specific pathogen-free housing conditions.

Collection of clinical samples. The use of human tissues was approved by the Institutional Review Board of Changzhou No. 2 People's Hospital. The formalin-fixed, paraffin-embedded tissue microarray of glioblastoma containing tissue samples collected from 164 patients were purchased from Shanghai Outdo Biotech Company (Table I). The written informed consents were collected from all patients.

Cell culture. U87 MG and U251 cells were grown in six-well plates using DMEM (Invitrogen: Thermo Fisher Scientific, Inc.) supplemented with $10 \% \mathrm{FBS}$ at $37^{\circ} \mathrm{C}$ in humidified air containing $5 \% \mathrm{CO}_{2}$. Cell culture media was replaced every $72 \mathrm{~h}$. The cells were subcultured at $80 \%$ confluence using $0.05 \%$ trypsin with $0.02 \%$ EDTA. The subsequent experiments were then performed on cells following a $24 \mathrm{~h}$ incubation in DMEM medium without FBS. All experiments were performed under serum-free conditions, in which the cells remained viable in a non-proliferating state.

Bioinformatics of KIF15 expression in glioma from a public database. The Cancer Genome Atlas (TCGA) database (https://cancergenome.nih.gov/) TCGA-GBM was utilized to collect the expression of KIF15 in 5 normal tissues and 169 GBM tissues.

Target gene RNA interference lentiviral vector preparation. Using the KIF15 gene as the template, the RNA interference targeting sequences (Pbr-13015/shKIF15-1, 5'-CAGGAT CGTTTGCTCTCAGAA-3'; Pbr-13016/shKIF15-2, 5'-AGG CAGCTAGAATTGGAATCA-3'; Pbr-00141/shKIF15-3, 5'-GCTGAAGTGAAGAGGCTCAAA-3') were designed and single-stranded DNA oligo were synthesized (Generay Biotech Co., Ltd.). The synthesized single-stranded DNA oligo was dissolved in an annealing buffer, using a water bath at $90^{\circ} \mathrm{C}$ for 15 min to form double-strand DNA. The BR-V-108 vector was linearized using AgeI and EcoRI. A $20 \mu \mathrm{l}$ reaction system was prepared according to the Fermentas T4 DNA Ligase instructions, and the double-stranded DNA oligo was ligated to the linearized vector $(100 \mathrm{ng} / \mu \mathrm{l})$. The ligation product was transferred into the prepared TOP10E. coli competent cells $(100 \mu \mathrm{l})$ with $500 \mu \mathrm{l}$ antibiotic-free lysogeny broth (LB) liquid medium and incubated at $37^{\circ} \mathrm{C}$ for $1 \mathrm{~h}$. A bacterial solution $(150 \mu \mathrm{l})$ was spread on LB solid medium containing Amp and cultured overnight in a $37^{\circ} \mathrm{C}$ incubator. A $20 \mu \mathrm{l}$ PCR reaction system was prepared, and a single colony was selected as a template with a sterile tip to perform PCR amplification. The correctly sequenced bacterial solution was transferred to $150 \mathrm{ml} \mathrm{LB}$ liquid medium containing Amp antibiotics, and cultured overnight at $37^{\circ} \mathrm{C}$. The plasmid was subsequently purified according to the EndoFree Maxi Plasmid Kit instructions (cat. no. DP117; Tiangen Biotech Co., Ltd.). The quality-qualified plasmids were transferred for virus packaging. Notably, the infection protocol included the temperature $\left(37^{\circ} \mathrm{C}\right)$, duration (18 h) and MOI (10) of infection. Subsequent experiments were performed after further culturing the cells for $72 \mathrm{~h}$.

Reverse transcription-quantitative $(R T-q) P C R$. Total RNA were isolated from U87 MG and U251 cells using TRIzol (Invitrogen; Thermo Fisher Scientific, Inc.) and Direct-zol ${ }^{\mathrm{TM}}$ RNA MiniPrep (Zymo Research), according to manufacturer's instructions. The RNA was quantified using NanoDrop 2000 (Thermo Fisher Scientific, Inc.). First-strand cDNA was obtained using $1 \mu \mathrm{g}$ total RNA, random primers and M-MLV reverse transcriptase (Promega Corporation). qPCR was conducted using AceQ qPCR SYBR Green Master mix (Vazyme). The $2^{-\Delta \Delta \mathrm{Cq}}$ method was utilized for the quantification of gene expression, with $\beta$-actin as an endogenous control. The following PCR primers were used: KIF15 forward, 5'-CTC TCACAGTTGAATGTCCTTG-3' and reverse, 5'-CTCCTT GTCAGCAGAATGAAG-3'; $\beta$-actin primers forward, 5'-CCT ATTTCCCATGATTCCTTCATA-3' and reverse, 5'-GTAATA CGGTTATCCACGCG-3'. The PCR protocol was: $3 \mathrm{~min}$ at $95^{\circ} \mathrm{C} ; 1 \mathrm{~min}$ at $94^{\circ} \mathrm{C}, 1 \mathrm{~min}$ at $60^{\circ} \mathrm{C}, 1 \mathrm{~min}$ at $72^{\circ} \mathrm{C}$ for 35 cycles; and $10 \mathrm{~min}$ at $72^{\circ} \mathrm{C}$. PCR products were separated on a $20 \mathrm{~g} / 1$ agarose gel stained with ethidium bromide and viewed under ultraviolet light.

Western blot analysis. In order to investigate the expression level of KIF15, cells were collected and lysed using RIPA lysis buffer (Cell Signal Technology, Inc.), including protease inhibitors, according to the manufacturer's instructions. The concentration of protein was determined using the BCA Protein Assay kit (cat. no. 23225; Thermo Fisher Scientific Pierce). Subsequently, the total cellular proteins were subjected to SDS-PAGE (10\% gel) for separation (20 $\mu \mathrm{g}$ protein per lane). After transferring to polyvinylidene difluoride (PVDF) membranes, blots were incubated with 5\% BSA in Tris-buffered 
Table I. Relationship between KIF15 expression and tumor characteristics in patients with glioma.

\begin{tabular}{lcccc}
\hline & & \multicolumn{2}{c}{ KIF15 } & \\
\cline { 3 - 4 } Features & No. of patients & Low & High & P-value \\
\cline { 3 - 4 } & & & & \\
All patients & 164 & 75 & 89 & \\
Age (years) & & & & $<0.0001$ \\
$\quad<41$ & 73 & 46 & 27 & \\
$\geq 41$ & 91 & 29 & 62 & \\
Sex & & & & 0.138 \\
Male & 106 & 53 & 53 & \\
Female & 58 & 22 & 36 & \\
Recurrence & & & & $<0.0001$ \\
Yes & 88 & 24 & 64 & \\
No & 76 & 51 & 25 & \\
Stage & & & & $<0.0001$ \\
I & 22 & 22 & 0 & \\
II & 71 & 61 & 10 & \\
III & 49 & 6 & 43 & \\
IV & 22 & 0 & 22 & \\
\hline
\end{tabular}

KIF15, kinesin-12.

saline containing $0.5 \%$ Tween-20 for $60 \mathrm{~min}$ and incubated overnight at $4{ }^{\circ} \mathrm{C}$ on a rocker with the following primary antibodies: KIF15 antibody (1:1,000; cat. no. FNab04551; Fine Test), GAPDH antibody (1:3,000; cat. no. AP0063; Bioworld), Bax antibody (1:1,000; cat. no. ab32503; Abcam), p21 antibody (1:400; cat. no. BM3990; Boster), Survivin antibody (1:500; cat. no. ab469; Abcam), MEK1/2 antibody (1:500; cat. no. ab178876; Abcam), p-MEK1/2 (1:500; cat. no. ab194754; Abcam), ERK1/2 (1:500; cat. no. 4695; CST) and p-ERK1/2 (1:500; cat. no. 4370; CST). Following three washes with TBST for $5 \mathrm{~min}$, the membranes were incubated with horseradish peroxidase (HRP)-conjugated goat anti-rabbit IgG polyclonal secondary antibody $(1: 3,000$; cat. no. A0208; Beyotime Institute of Biotechnology) at room temperature for $1 \mathrm{~h}$. Amersham ECL + plus ${ }^{\mathrm{TM}}$ western blotting system kit (cat. no. RPN3352; GE Healthcare) was used for color developing. Each membrane was visualized using the ECL-Plus $^{\mathrm{TM}}$ Western blotting system (GE Healthcare Life Sciences), and proteins were detected with an X-ray imaging analyzer (Kodak). Densitometric analysis was performed using ImageJ (version 1.8.0; National Institutes of Health).

MTT assay. An MTT assay was applied to analyze in vitro cell viability. Following the trypsinization of cells in the logarithmic growth phase, glioma cells $(2,000$ cells/well) were seeded into a 96-well (100 $\mu \mathrm{l} /$ well) (cat. no. 3599; Corning Inc.) overnight. MTT solution (20 $\mu \mathrm{l} / \mathrm{well}, 5 \mathrm{mg} / \mathrm{ml}$; cat. no. JT343; Genview) was added to cells and incubated for $4 \mathrm{~h}$. DMSO (100 $\mu \mathrm{l} /$ well) was added to dissolve the formazan crystals. Absorbance values were measured at $490 \mathrm{~nm}$ using a microplate reader (cat. no. M2009PR; Tecan Group, Ltd.) after 24,
$48,72,96$, and $120 \mathrm{~h}$ of growth; $570 \mathrm{~nm}$ was used as the reference wavelength. The cell viability ratio was calculated using the following formula: cell viability $(\%)=\mathrm{OD}$ (treated)/OD (control) $\mathrm{x} 100 \%$.

Apoptotic assay. Lentivirus-infected cells were seeded in $6 \mathrm{~cm}$ dishes. The cells were subsequently digested with trypsin and resuspended in the same medium, then $10 \mu 1$ Annexin $\mathrm{V}$-allophycocyanin (APC) was added for staining the cells for 15 min in a dark at room temperature. Cell apoptosis analysis was performed using an Annexin V-Allophycocyanin/Propidium Iodide kit (eBioscience; Thermo Fisher Scientific, Inc.) The apoptotic rate of cells was measured using a FACScan analyzer (Merck KGaA). The results were visualized using GuavaSoft software (version 3.1.1; EMD Millipore).

Detection of cell cycle by fluorescence-activated cells sorting (FACS). Cells in the exponential growth phase were collected. The cells were subsequently washed with cold PBS and fixed in $950 \mu \mathrm{l}$ of cold $70 \%$ ethanol for $1 \mathrm{~h}$. Finally, cells were stained by propidium iodide (PI) and kept away from light for 10-15 min at room temperature. The cell cycle was detected by BD FACSCalibur flow cytometer (BD Biosciences) with the throughput of cells $\sim 200-350$ cells.

Immunohistochemistry analysis. The expression of KIF15 was detected by immunohistochemistry in glioblastoma tissues. Tumor and para-carcinoma tissue sections from glioblastoma patients were deparaffinized. Following citrate antigen repair and blocking by $3 \% \mathrm{H}_{2} \mathrm{O}_{2}$ for $10 \mathrm{~min}$ at room temperature, the samples were incubated with the KIF15 antibody (1:50; Fine Test) at $4{ }^{\circ} \mathrm{C}$ overnight in an incubator. The sections were subsequently incubated with goat anti-rabbit IgG H\&L horseradish peroxidase (HRP)-conjugated secondary antibody (1:400; cat. no. ab6721; Abcam) at $37^{\circ} \mathrm{C}$ for $1 \mathrm{~h}$. Images were captured by a light microscope (Olympus Corp.). Ki-67 antibody (1:100; cat. no. ab16667; Abcam), Bax antibody (1:250; cat. no. ab32503; Abcam), p21 antibody (1:50; cat. no. BM3990; BOSTER) and Survivin antibody (1:100; cat. no. ab469; Abcam) were used in the immunohistochemistry analysis of tumor sections removed from mice models.

Caspase 3/7 activity assay. Cell apoptosis was also quantified by the Caspase-Glo ${ }^{\circledR}$ 3/7 assay kit (Promega Corp.), according to the manufacturer's protocol.

Ethics approval and tumor-bearing animal model. All the animal experimental protocols were approved by the Ethics committee at The Affiliated Changzhou No. 2 People's Hospital of Nanjing Medical University. BALB/c male nude mice (10-15 g, 6 weeks old) were cultured in SPF-class housing in the laboratory with a 12 day/night environment and fed with a standard diet. U87 MG cells $\left(5 \times 10^{6}\right)$, transfected with shKIF5 or shCtrl, were implanted into the right underarm of mice (BALB/c male nude mice) subcutaneously. Volumes of the xenograft tumors were measured by calipers after $7,13,19$, 22 and 28 days post-infection. Although the humane endpoint in our protocol was a tumor $>15 \mathrm{~mm}$, all mice were sacrificed before reaching the humane endpoint. After 28 days of culture, the mice were sacrificed by injecting $1 \%$ pentobarbital sodium 
(100 $\mathrm{mg} / \mathrm{kg}$ body weight), and the tumors were collected for the measurement of weight and for immunohistochemistry (IHC) analysis.

Human apoptosis antibody array. The expression profiles of a series of apoptosis-associated proteins induced by KIF15 knockdown in U87MG cells were detected by human apoptosis antibody array (RayBiotech), according to manufacturer's instructions. Briefly, the array membrane was placed into a dish and cell lysates were added to each well for incubation at $4^{\circ} \mathrm{C}$ with gentle shaking overnight. The membranes were washed and then incubated with lyophilized biotinylated antibodies for $1 \mathrm{~h}$ on a rocking platform shaker. After washing away the excess molecules, the membranes were further incubated with horseradish peroxidase-conjugated streptavidin for $30 \mathrm{~min}$. The expression levels of proteins were analyzed using the Gel-Pro Analyzer software (version 6.3; Media Cybernetics).

Statistical analysis. All experiments were carried out in at least 3 independent experiments. Data were expressed as the mean \pm standard deviation for continuous variables and analyzed using GraphPad Prism 6 software (GraphPad Software, Inc.). Student's t-test and $\chi^{2}$ test were used to analyze the statistical difference. Statistical analysis between multiple groups was performed using ANOVA followed by Tukey's HSD post hoc test. The survival analysis was performed by the Kaplan-Meier method and analyzed by the log-rank test. $\mathrm{P}<0.05$ was considered to indicate a statistically significant difference.

\section{Results}

KIF15 is upregulated in glioma tissues and is associated with poor prognosis. In order to investigate the role of KIF15 in the prognosis of glioma, clinical specimens of glioma tissues and the corresponding follow-up information were collected. The protein expression level of KIF15 was detected by IHC analysis in glioma tissues at different pathological stages. The results revealed that the expression of KIF15 was significantly higher at the later stage (Fig. 1A). RNA-seq data mining of The Cancer Genome Atlas (TCGA) database confirmed that KIF15 expression was significantly increased in glioma tissues compared with normal tissues (Fig. 1B). Furthermore, high expression of KIF15 in glioma tissues was associated with older age, late pathological stage and risk of recurrence (Table I). Moreover, the Kaplan-Meier survival analysis revealed that patients with relatively high expression of KIF15 suffered from notably shorter overall survival, as well as poor prognosis (Fig. 1C). The aforementioned results indicated the involvement of KIF15 in the development and progression of glioma and its potential role as a prognostic indicator.

Construction of KIF15 KD cell models. In order to clarify the role of KIF15 in glioma further, two glioma cell lines U87 MG and U251 were selected for the construction of KIF15-knockdown (KD) cell models. Artificially prepared lentivirus plasmid shKIF15, which expressed 3 different RNAi sequences (Pbr-03015, Pbr-13016 and Pbr-00141) for silencing
KIF15, were transfected into cells to downregulate the levels of KIF15 expression. The cells transfected with the corresponding empty vector were used as the negative control (shCtrl). The transduction efficiency in both U87 MG and U251 cells was assessed through the detection of green fluorescent protein (GFP), which was tagged on the lentivirus vector; $>80 \%$ efficiency was revealed in both cell lines for both the shCtrl and shKIF15 groups (Fig. 1D). Subsequently, through the detection of mRNA and protein levels of KIF15 in each group by qPCR and western blotting, respectively, Pbr-00141 was identified as the most efficient sequence for KIF15 knockdown in both cell lines; and was thus used in all subsequent experiments for the shKIF15 group (Fig. 1E and F). In summary, given the upregulated expression level of KIF15 in clinical specimens, the KIF15-KD cell models were successfully constructed for the subsequent studies.

Silencing of KIF15 inhibits cell proliferation and stemness, induces cell apoptosis and affects the cell cycle in glioma cells. The effect of KIF15 KD on the functions of glioma cells was evaluated. The results of the MTT assay revealed that the downregulation of KIF15 in U87 MG and U251 cells significantly inhibited cell proliferation (Fig. 2A). Moreover, flow cytometric analysis was performed to explore the effect of KIF15 on cell apoptosis in U87 MG and U251 cells. As revealed in Fig. 2B, the silencing of KIF15 notably enhanced cell apoptosis in both cell lines. The apoptosis percentages for KIF15-KD U87 MG and KIF15-KD U251 cells were 5- and 3.8-fold higher compared with the shCtrl groups, respectively. Moreover, KIF15 KD-induced cell apoptosis was also assessed by the detection of caspase 3/7 activity (Fig. 2C). Furthermore, as revealed in Fig. 2D, cell cycle analysis indicated an increased percentage of cells in the $\mathrm{G}_{2}$ phase in the KIF15-KD groups for both cell lines; this demonstrated the $\mathrm{G}_{2}$-arresting ability of KIF15 KD in glioma cells. Furthermore, the expression levels of stemness-associated proteins OCT4 and SOX2 were both inhibited in U87 MG and U251 cells following the silencing of KIF15 (Fig. 2E). Overall, the silencing of KIF15 inhibited cell proliferation and stemness, induced cell apoptosis and promoted $\mathrm{G}_{2}$-arrest in glioma cells.

Silencing of KIF15 impairs tumor growth in vivo. The ability of KIF15 KD to impair tumor growth was assessed in vivo. Therefore, a mouse xenograft model was constructed by subcutaneously injecting U87 MG cells with or without KIF15 KD into nude mice and monitoring tumor growth. The weight and volume of the tumors removed from mice in the shKIF15 group were significantly lower compared with the shCtrl group (time-point 1: 7 days; 2: 13 days; 3: 19 days; 4: 22 days; 5: 28 days following inoculation; $\mathrm{P}<0.001$; Fig. 3A-C). Moreover, the tumors formed in the shKIF15 group displayed lower Ki-67 index compared with tumors removed from the shCtrl group, as detected by IHC analysis (Fig. 3D). Overall, these results indicated that the silencing of KIF15 resulted in impaired tumorigenicity of glioma cells in vivo, which was consistent with the data obtained in the proliferation and apoptosis assays in vitro.

Mechanistic study of KIF15 in glioma. In order to explore the potential mechanism of the regulatory ability of KIF15 
A

Stage I

Stage II

Stage IV
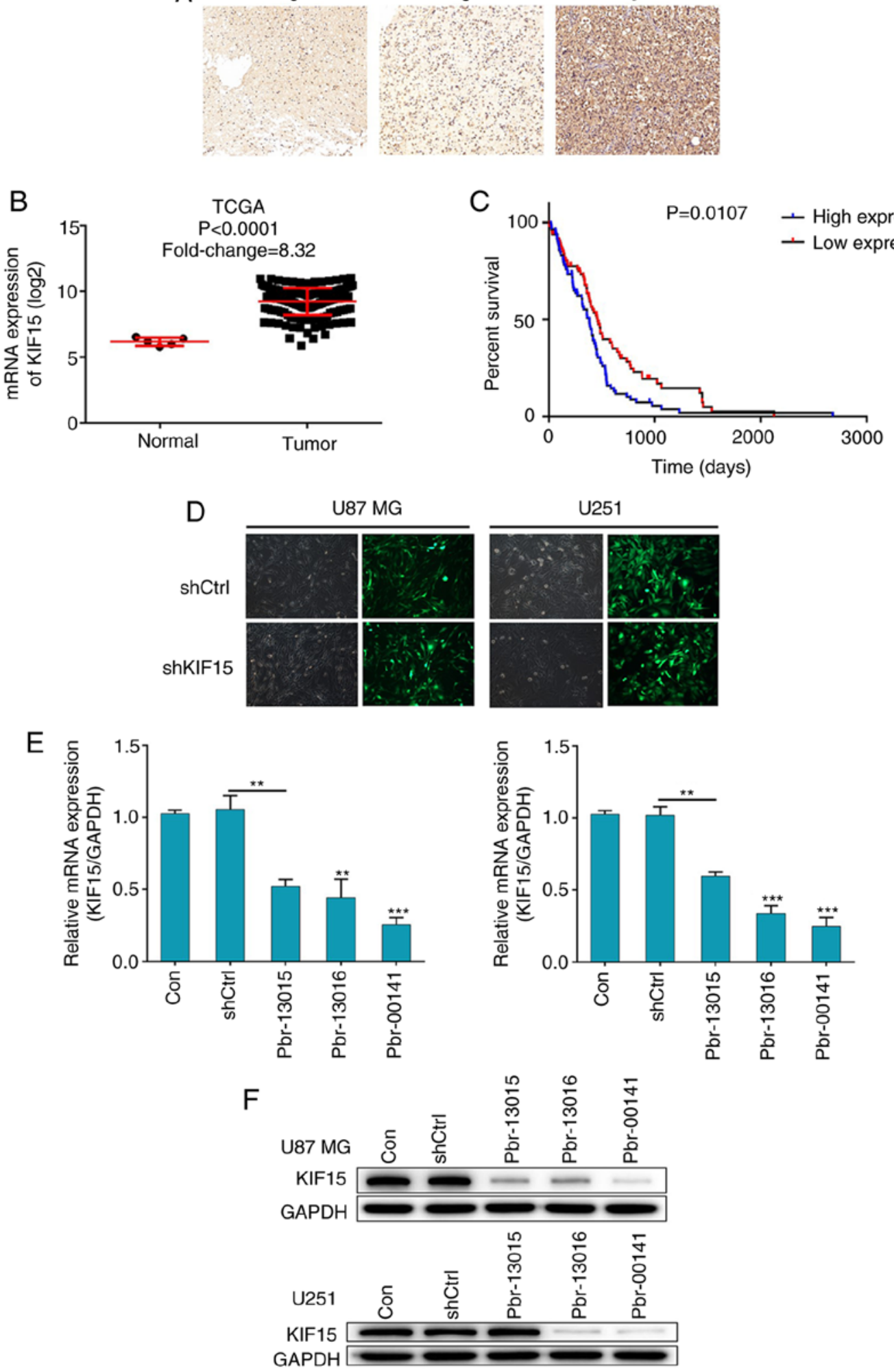

Figure 1. High expression of KIF15 in glioma tissues and construction of KIF15-KD cell models. (A) Expression levels of KIF15 in glioma tissues, infiltrated carcinoma tissues and normal tissues were detected by IHC staining (magnification x200). (B) KIF15 mRNA expression in TCGA RNA-seq database. (C) Overall survival of the patients displaying high or low KIF15 expression. (D) Infection efficiencies for U87 MG and U251 cell lines were evaluated by detection of green fluorescent protein $72 \mathrm{~h}$ post-infection (magnification $\mathrm{x} 200$ ). (E and F) The validity and efficiency of KIF15 KD were evaluated by (E) qPCR and (F) western blot analysis (Con means the group of cells without transfection), respectively. ${ }^{* *} \mathrm{P}<0.01,{ }^{* * *} \mathrm{P}<0.001$. KIF15, kinesin- 12 ; KD, knockdown; IHC, immunohistochemistry; TCGA, The Cancer Genome Atlas.

in glioma, human apoptosis antibody array was performed to identify the differentially expressed proteins induced by KIF15 KD in U87 MG cells compared with the shCtrl group.
The upregulated expression of CD40 and CD40L in shKIF15 group revealed in Fig. 4A, indicated the potential participation of the CD40/CD40L signaling pathway in KIF15-induced 
A

U87 MG

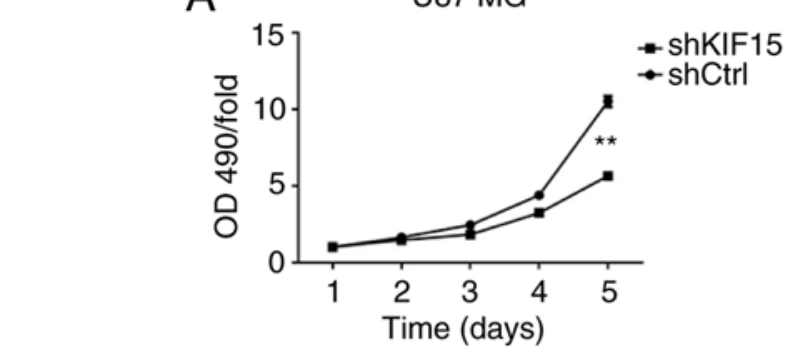

U251

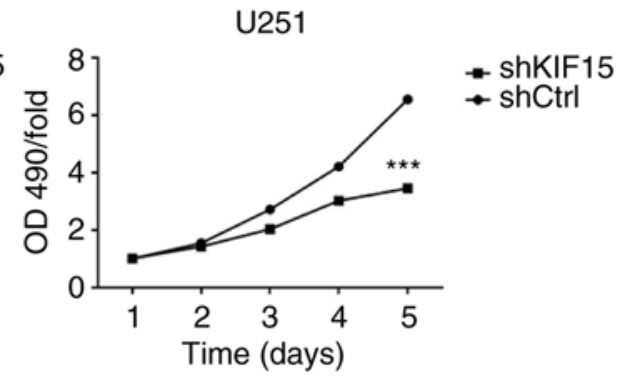

B
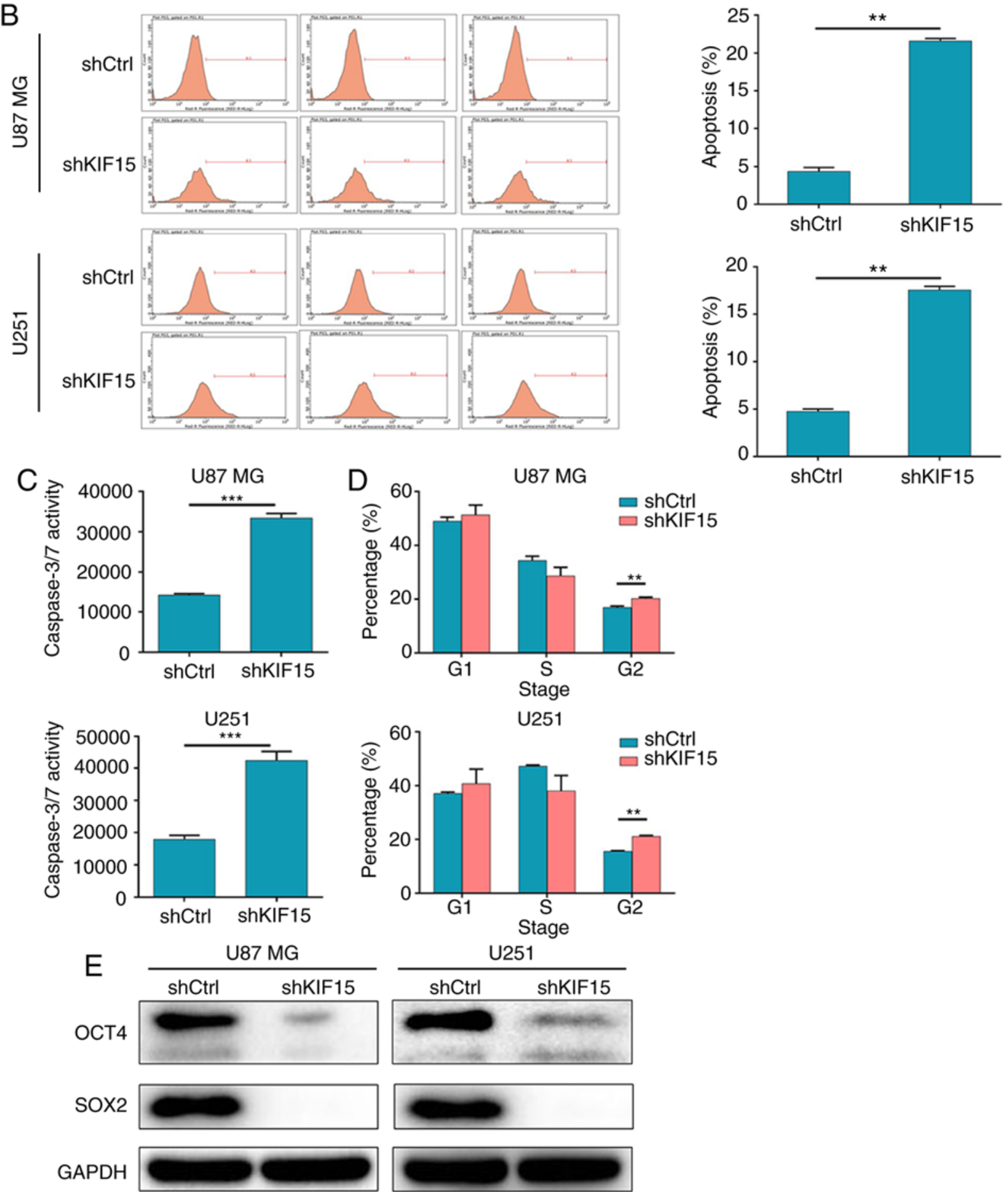

Figure 2. Knockdown of KIF15 inhibits cell proliferation and stemness and induces cell apoptosis. (A) An MTT assay was used to evaluate the cell proliferation of U87 MG and U251 cells with KIF15 KD and compared with negative control. (B) Flow cytometric analysis was employed to detect the percentage of apoptotic cells in U87 MG and U251 cells with KIF15 KD and the negative control. (C) The detection of Caspase-3/7 activity in U87 MG and U251 cells. (D) Cell cycle analysis of U87 MG and U251 cells with KIF15 KD and the negative control and the percentage of cells in cell cycle phases G1, S and G2. (E) Western blot analysis was performed to detect the expression levels of stemness-related proteins OCT4 and SOX2. Data are presented as the mean \pm SD $(\mathrm{n} \geq 3) .{ }^{* *} \mathrm{P}<0.01,{ }^{* * *} \mathrm{P}<0.001$. KIF15, kinesin-12; KD, knockdown. 


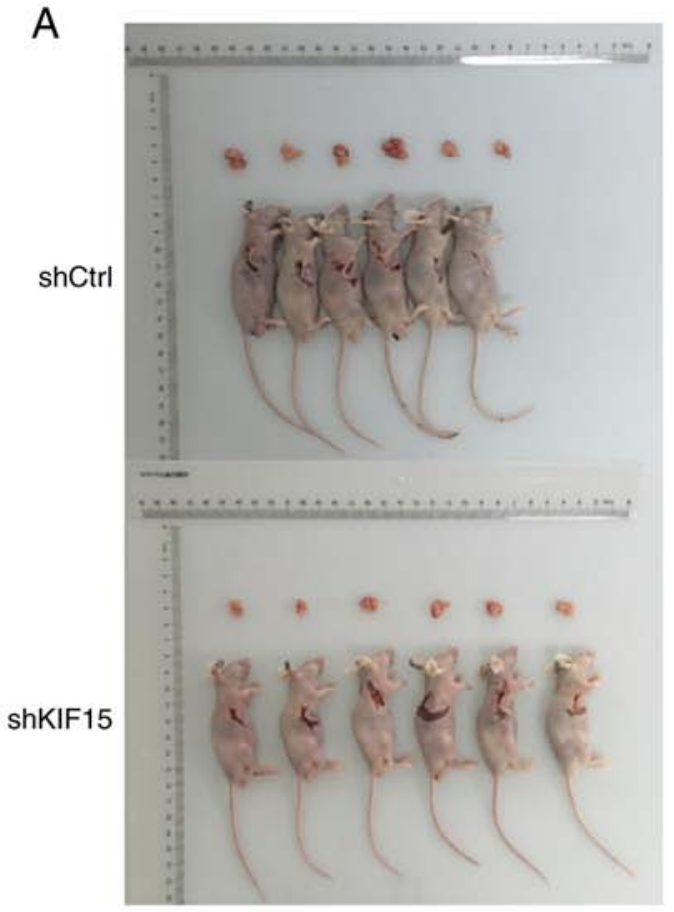

D

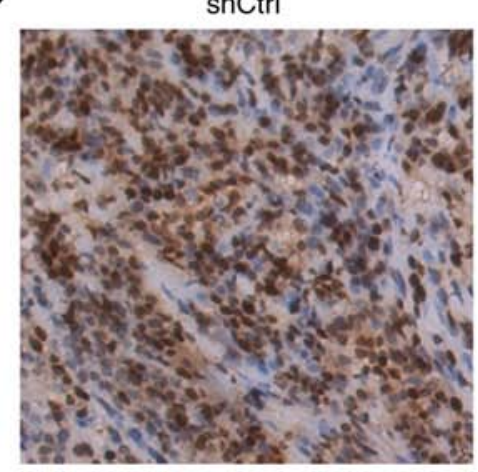

B

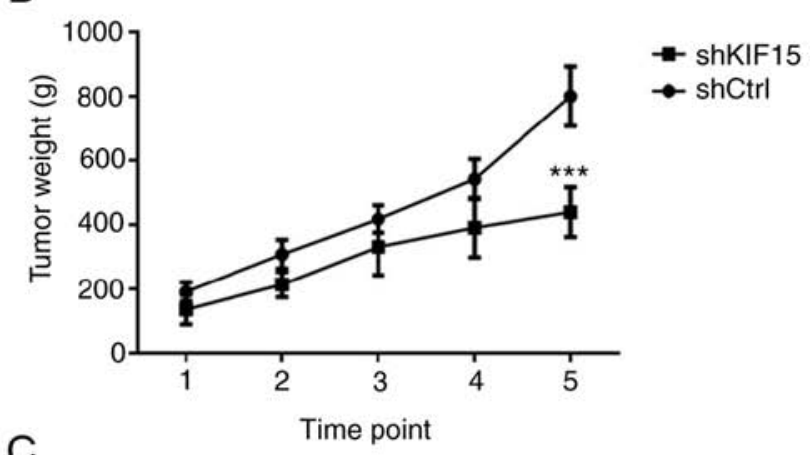

C

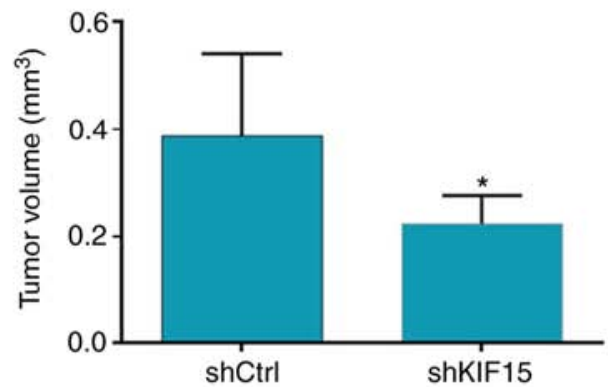

ShKIF15

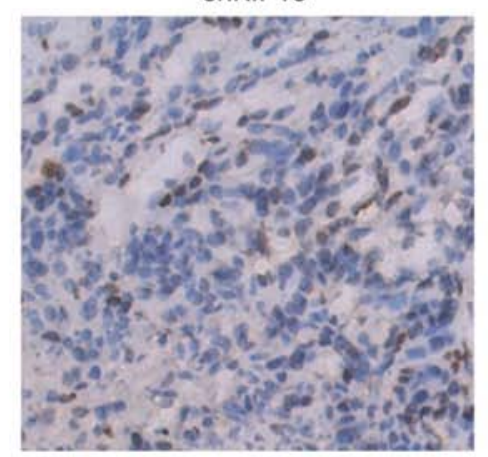

Figure 3. Knockdown of KIF15 inhibits the tumor growth in vivo. (A) The tumor images obtained from mice in the shCtrl group and shKIF15 group. (B) The average weight of tumors in shCtrl group and shKIF15 group. (C) The volume of tumors in shCtrl group and shKIF15 group was measured at 7, 13, 19, 22, 28 (time-point 1: 7 days; 2: 13 days; 3: 19 days; $4: 22$ days; 5: 28 days days post-injection. (D) The expression of Ki-67 in sections of tumors was detected by IHC. Data are presented as the mean $\pm \mathrm{SD}(\mathrm{n} \geq 3)$. $\mathrm{P}<0.05,{ }^{* * * *} \mathrm{P}<0.001$. KIF15, kinesin- 12 .

inhibition in glioma. Moreover, several proteins associated with apoptosis and cell cycle were selected for verification in U251 cells. As revealed in Fig. 4B, the protein expression of pro-apoptotic proteins Bax and p21 were also upregulated in U251 cells with KIF15 KD, whereas the anti-apoptosis protein Survivin was downregulated; thus demonstrating the ability of KIF15 KD to induce cell apoptosis, which is consistent with the aforementioned cellular experiments. In addition, the upregulated expression levels of Bax and p21, and downregulated expression of Survivin detected by IHC were also observed in the tumor tissues from mice in the shKIF15 group (Fig. 4C). Conversely, the effects of KIF15 on the activation of the MEK/ERK signaling pathway were investigated, based on the detection of MEK1/2, p-MEK1/2, ERK1/2, p-ERK1/2 by western blot analysis. The results indicated that, the expression of p-MEK1/2 and p-ERK1/2 was downregulated by KIF15 $\mathrm{KD}$, whereas the expression of MEK $1 / 2$ and ERK $1 / 2$ remained unchanged, indicating the inhibition of the MEK/ERK signaling pathway by KIF15 knockdown (Fig. 4D).

\section{Discussion}

Glioma is the most common primary intracranial malignant tumor in adults, and also the most common intracranial malignancy in children $(3,24)$. It has the characteristics of rapid growth, strong infiltration and high recurrence rate (25). At present, among the various treatment strategies for cancer, the most commonly used treatment for gliomas is the combination of surgical resection and radiotherapy/chemotherapy. However, the median survival period of patients with glioma was revealed to be only 12 to 18 months, and most patients fail to survive the next 2 years following diagnosis (26). In recent years, the development of molecular biology has deepened the understanding of the molecular characteristics of malignant glioma and promoted the discovery of some glioma markers. For example, the epidermal growth factor receptor signaling pathway plays an important role in the pathogenesis, proliferation, invasion and mediation of drug resistance in glioma, and its expression increases with the 
A shCtrl

ShKIF15
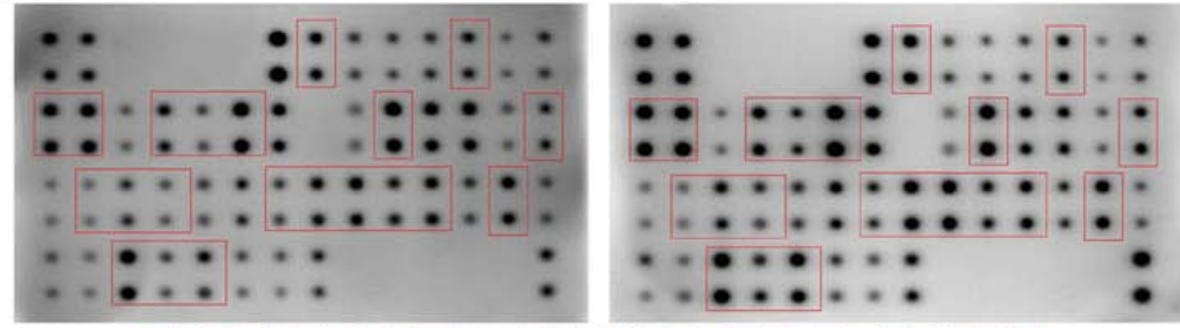

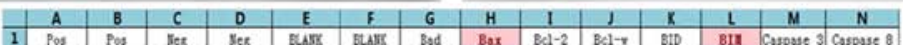

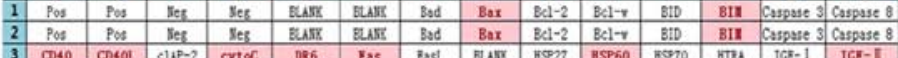

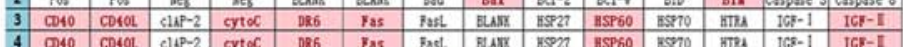

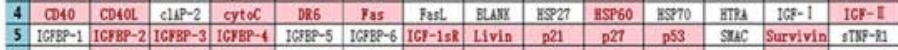

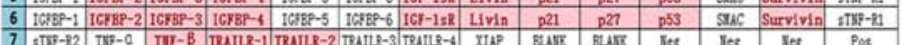

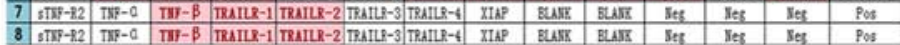

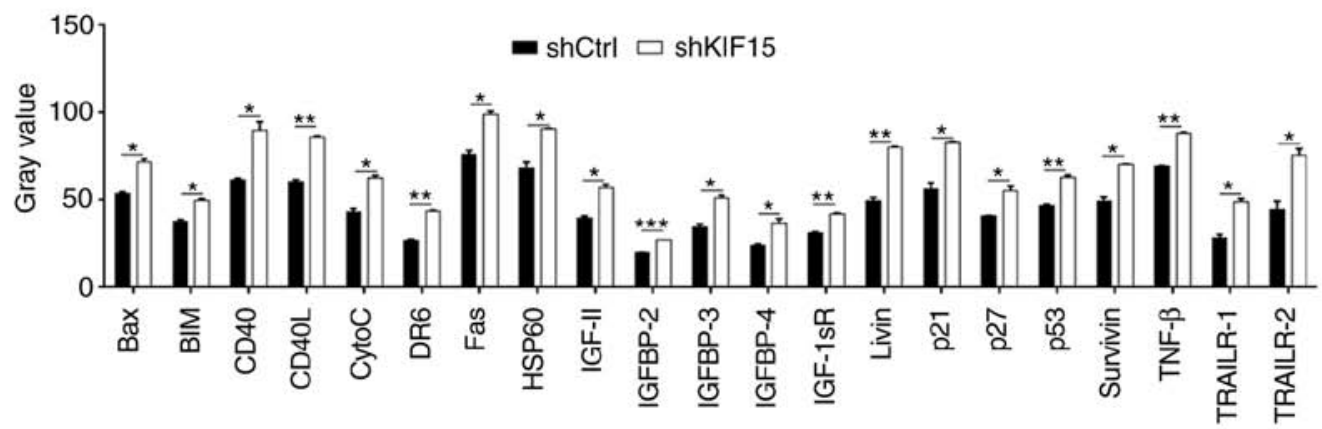

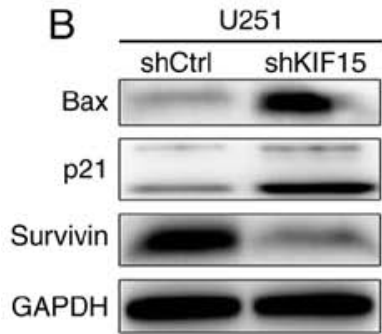

C
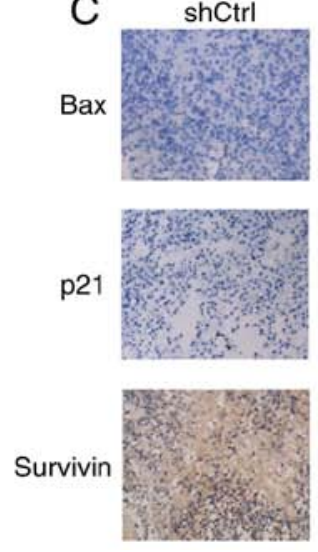
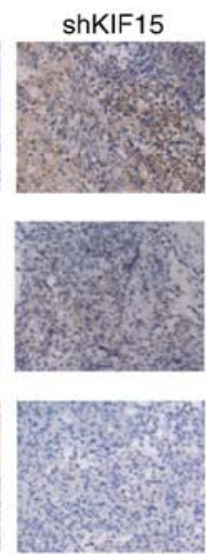
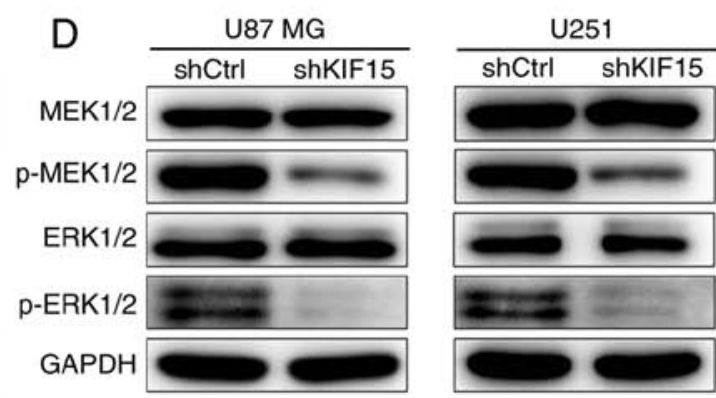

Figure 4. Mechanism study of KIF15 KD in glioma. (A) Human apoptosis antibody array analysis was performed and analyzed in U87 MG cells with or without KIF15 KD. (B) The protein expression levels of Bax, p21 and Survivin in U251 cells were detected by western blot analysis. (C) The expression levels of Bax, p21 and Survivin were detected by IHC in tumor sections of a mouse xenograft model (magnification, x200). (D) The expression levels of MEK1/2, p-MEK1/2, ERK1/2, p-ERK1/2 were detected by western blot analysis in U87 MG and U251 cells with or without KIF15 KD. ${ }^{*} \mathrm{P}<0.05,{ }^{* * *} \mathrm{P}<0.01,{ }^{* * * *} \mathrm{P}<0.001$. KIF15, kinesin-12; KD, knockdown.

increasing tumor grade (27). Moreover, Yan et al (28) reported that miR-96 could promote the development and progression of glioma, through the enhancement of the activation of the Wnt/ $\beta$-catenin signaling pathway. As a member of the Eph subfamily screened from the human epithelial keratinocyte cDNA library by Wykosky et al (29), EphA2 was specifically reported to be highly expressed in nearly $90 \%$ of GBM tissues and cell lines, whereas its expression remains normal in brain tissues. Furthermore, several proteins, such as YKL-40 and CD133 and long non-coding (lnc)RNAs such as lncRNA MALAT1, have been demonstrated to be involved in the promotion or suppression of glioma $(30,31)$. However, more accurate and specific biomarkers for glioma still need to be explored for the better understanding of its mechanism and for the development of novel treatment strategies.
Kinesin is a protein superfamily that plays an important role in eukaryotic intracellular trafficking and cell division (10). Kinesins and the intracellular transport of substances participated by kinesins play an important role in maintaining the basic functions of cells (13). Previous studies have revealed that kinesins were directly related to several types of diseases such as neurodegenerative diseases. Notably, accumulating evidence revealed that some members of the kinesin superfamily were associated with human malignancies. Wang et al (32) reported that the downregulation of KIF2A could inhibit the proliferation and migration of breast cancer cells and has the potential to be used as an independent prognostic marker of outcome in breast cancer. The expression of KIF23 was also reported to be associated with the prognosis of patients undergoing lung cancer surgery and has the potential as a therapeutic target for 
the development of lung cancer treatment (33). Furthermore, some kinesin members were also revealed to be involved in glioma. For example, the mitotic kinesin KIF11 could promote the proliferation, invasion and self-renewal of GBM (34-36). Chen et al (37) revealed that KIF1B could be a target for anti-invasive therapies of glioma due to its ability to promote migration and invasion in glioma, through the cell surface localization of MT1-MMP.

KIF15, also named kinesin-12, is a microtubule-dependent plus-end-directed motor protein, which is best known for its role in mitosis. A study by Liu et al (38) revealed that KIF15 was highly expressed in both the cortex and ganglia at embryonic stages, whereas its expression diminished progressively with the development of neurons. Furthermore, KIF15 was recognized as a modulator of axonal development (39). Moreover, the structure and function of KIF15 was also studied in recent years due to the speculation that it may be a useful target for cancer therapy. Studies have revealed that KIF15 plays an important role in the maintenance of spindle bipolarity and is a breast cancer tumor antigen (40). Recently, KIF15 was reported to be overexpressed in both breast cancer and lung adenocarcinoma, and is potentially involved in the regulation of the cell cycle $(41,42)$. Moreover, Wang et al $(22)$ demonstrated that KIF15 could promote pancreatic cancer cell proliferation via the regulation of the MEK/ERK pathway. To the best of our knowledge, the potential of KIF15 as a target for anti-growth therapies in glioma has not been reported and is still unclear.

In the present study, clinical specimens were collected to detect the expression of KIF15 by IHC, which revealed relatively high KIF15 expression at the later-stage glioma tissues. The high expression of KIF15 in glioma tissues compared with normal tissues was demonstrated by the RNA-seq data of TCGA. Moreover, the statistical analysis revealed that patients with high KIF15 expression were associated with older age, late pathological stage, high recurrence risk and shorter survival period. The results obtained from the clinical samples indicated the potential association between KIF15 expression and the development and prognosis of glioma.

To verify and further explore the role of KIF15 in the development and progression of glioma, U87 MG and U251 cell lines were selected to construct KIF15-KD cell models. According to the different capabilities of the 3 shRNAs designed for silencing KIF15 to knockdown KIF15, inhibit cell proliferation and promote cell apoptosis (data not shown), the most efficient one was screened for all in vitro and in vivo studies. The subsequent studies revealed that the silencing of KIF15 inhibited cell proliferation, induced cell apoptosis and promoted the arrest of glioma cells at the $\mathrm{G}_{2}$ phase. Notably, the stemness of glioma cells, which was demonstrated to be the fundamental source of drug resistance and recurrence of gliomas, was also significantly inhibited by KIF15 KD, revealed by the downregulation of OCT4 and SOX2 expression levels. In addition, the generally similar expression of the internal reference (GAPDH) also demonstrated that the downregulation of OCT4 and SOX2 did not result from enhanced cell death. Moreover, the mouse xenograft model revealed that KIF15 KD inhibited tumor growth and the expression of Ki-67 in vivo. Moreover, a human apoptosis antibody array was performed, which revealed the potential role of the CD40/CD40L signaling pathways. Furthermore, the changes in the expression of various apoptosis-associated proteins, including Bax, p21 and Survivin, in the shKIF15 group were verified in both the cellular and animal experiments, and were consistent with the results of cell apoptosis. Moreover, consistent with the reported ability of KIF15 to regulate the MEK/ERK signaling pathway (22), the present study demonstrated that the knockdown of KIF15 markedly downregulated the expression of p-MEK1/2 and p-ERK1/2, as well as deactivated the MEK/ERK signaling pathway. The aforementioned results indicated the involvement of KIF15 in the development and progression of glioma.

In conclusion, the present study indicated that KIF15 may act as tumor promoter in glioma and could be a future therapeutic target and prognostic indicator. Further studies focusing on the regulatory mechanism of KIF15 in glioma could benefit the better understanding of glioma carcinogenesis.

\section{Acknowledgements}

Not applicable.

\section{Funding}

The present study was financially supported by the National Natural Science Foundation of China (No. 81302181), the Jiangsu Provincial Medical Youth Talent Programme and Key Research and Development Program of Jiangsu Province (BE2019652).

\section{Availability of data and materials}

The datasets used and/or analyzed during the current study are available from the corresponding author on reasonable request.

\section{Authors' contributions}

FL made substantial contributions to the concept and design of the present study. QW, WH and CQ conducted the experiments. CQ and BH conducted data analysis. QW, WH and FL produced the manuscript. All authors read and approved the final manuscript.

\section{Ethics approval and consent to participate}

The use of human tissues was approved by the Institutional Review Board of Changzhou No. 2 People's Hospital. The written informed consents were collected from all patients. All the animal experimental protocols were approved by the Ethics committee at The Affiliated Changzhou No. 2 People's Hospital of Nanjing Medical University.

\section{Patient consent for publication}

Not applicable.

\section{Competing interests}

The authors have no conflicts of interest to disclose. 


\section{References}

1. Weller M, Pfister SM, Wick W, Hegi ME, Reifenberger G and Stupp R: Molecular neuro-oncology in clinical practice: A new horizon. Lancet Oncol 14: e370-e379, 2013.

2. Maher EA, Furnari FB, Bachoo RM, Rowitch DH, Louis DN, Cavenee WK and DePinho RA: Malignant glioma: Genetics and biology of a grave matter. Genes Dev 15: 1311-1333, 2001.

3. Wen PY and Kesari S: Malignant gliomas in adults. New Engl J Med 359: 492-507, 2008.

4. Nutt CL, Mani DR, Betensky RA, Tamayo P, Cairncross JG, Ladd C, Pohl U,Hartmann C, Mclaughlin ME, Batchelor TT, et al: Gene expression-based classification of malignant gliomas correlates better with survival than histological classification. Cancer Res 63: 1602-1607, 2003

5. Nayak L and Reardon DA: High-Grade gliomas. Continuum (Minneap Minn) 23: 1548-1563, 2017.

6. Stupp R, Mason WP, van den Bent MJ, Weller M, Fisher B, Taphoorn MJ, Belanger K, Brandes AA, Marosi C, Bogdahn U, et al: Radiotherapy plus concomitant and adjuvant temozolomide for glioblastoma. New Engl J Med 352: 987-996, 2005.

7. Khasraw $\mathrm{M}$ and Lassman AB: Advances in the treatment of malignant gliomas. Curr Oncol Rep 12: 26-33, 2010.

8. Cohen AL and Colman H: Glioma biology and molecular markers. Cancer Treat Res 163: 15-30, 2015.

9. Kros JM, Mustafa DM, Dekker LJ, Sillevis Smitt PA, Luider TM and Zheng PP: Circulating glioma biomarkers. Neuro Oncol 17: 343-360, 2015.

10. Hirokawa $\mathrm{N}$ and Tanaka Y: Kinesin superfamily proteins (KIFs): Various functions and their relevance for important phenomena in life and diseases. Exp Cell Res 334: 16-25, 2015.

11. Hirokawa N, Bloom GS and Vallee RB: Cytoskeletal architecture and immunocytochemical localization of microtubule-associated proteins in regions of axons associated with rapid axonal transport: The beta,beta'-iminodipropionitrile-intoxicated axon as a model system. J Cell Biol 101: 227-239, 1985.

12. Hirokawa N, Niwa S and Tanaka Y: Molecular motors in neurons: Transport mechanisms and roles in brain function, development, and disease. Neuron 68: 610-638, 2010.

13. Okada Y, Yamazaki H, Sekine-Aizawa Y and Hirokawa N: The neuron-specific kinesin superfamily protein KIF1A is a unique monomeric motor for anterograde axonal transport of synaptic vesicle precursors. Cell 81: 769-780, 1995.

14. Ari C, Borysov SI, Wu J, Padmanabhan J and Potter H: Alzheimer amyloid beta inhibition of Eg5/kinesin 5 reduces neurotrophin and/or transmitter receptor function. Neurobiol Aging 35: 1839-1849, 2014.

15. Mcguire JR, Rong J, Li SH and Li XJ: Interaction of huntingtinassociated protein-1 with kinesin light chain: Implications in intracellular trafficking in neurons. J Biol Chem 281: 3552-3559, 2006.

16. Yang W, Tanaka Y, Bundo M and Hirokawa N: Antioxidant signaling involving the microtubule motor KIF12 is an intracellular target of nutrition excess in beta cells. Dev Cell 31: 202-214, 2014.

17. Mrug M, Li R, Cui X, Schoeb TR, Churchill GA and Guay-Woodford LM: Kinesin family member 12 is a candidate polycystic kidney disease modifier in the cpk mouse. J Am Soc Nephrol 16: 905-916, 2005.

18. Mazumdar M, Lee JH, Sengupta K, Ried T, Rane S and Misteli T: Tumor formation via loss of a molecular motor protein. Curr Biol 16: 1559-1564, 2006

19. Chandrasekaran G, Tátrai P and Gergely F: Hitting the brakes: Targeting microtubule motors in cancer. Br J Cancer 113 693-698, 2015

20. Wang W, Shi Y, Li J, Cui W and Yang B: Up-Regulation of KIF14 is a predictor of poor survival and a novel prognostic biomarker of chemoresistance to paclitaxel treatment in cervical cancer. Bioscience Rep 36: e315, 2016.

21. Sturgill EG, Norris SR, Guo Y and Ohi R: Kinesin-5 inhibitor resistance is driven by kinesin-12. J Cell Biol 213: 213-227, 2016.

22. Wang J, Guo X, Xie C and Jiang J: KIF15 promotes pancreatic cancer proliferation via the MEK-ERK signalling pathway. Brit J Cancer 117: 245-255, 2017.

23. Milic B, Chakraborty A, Han K, Bassik MC and Block SM: KIF15 nanomechanics and kinesin inhibitors, with implications for cancer chemotherapeutics. Proc Natl Acad Sci USA 115: E4613-E4622, 2018.
24. Pollack IF, Finkelstein SD, Woods J, Burnham J, Holmes EJ, Hamilton RL, Yates AJ, Boyett JM, Finlay JL, Sposto R, et al: Expression of p53 and prognosis in children with malignant gliomas. New Engl J Med 346: 420-427, 2002.

25. Omuro A and Deangelis LM: Glioblastoma and other malignant gliomas: A clinical review. JAMA 310: 1842-1850, 2013.

26. Tafani M, De Vito M, Frati A, Pellegrini L, De Santis E, Sette G, Eramo A, Sale P, Mari E, Santoro A, et al: Pro-inflammatory gene expression in solid glioblastoma microenvironment and in hypoxic stem cells from human glioblastoma. J Neuroinflamm 8: 32, 2011.

27. Sampson JH, Choi BD, Sanchez-Perez L, Suryadevara CM, Snyder DJ, Flores CT, Schmittling RJ, Nair SK, Reap EA, Norberg PK, et al: EGFRvIII mCAR-modified T-cell therapy cures mice with established intracerebral glioma and generates host immunity against tumor-antigen loss. Clin Cancer Res 20: 972-984, 2016.

28. Yan Z, Wang J, Wang C, Jiao Y, Qi W and Che S: MiR-96/HBP1/ Wnt/ $\beta$-catenin regulatory circuitry promotes glioma growth. FEBS Lett 588: 3038-3046, 2016.

29. Wykosky J, Gibo DM, Stanton C and Debinski W: Interleukin-13 receptor alpha 2, EphA2, and Fos-related antigen 1 as molecular denominators of high-grade astrocytomas and specific targets for combinatorial therapy. Clin Cancer Res 14: 199-208, 2008.

30. Han Y, Wu Z, Wu T, Huang Y, Cheng Z, Li X, Sun T, Xie X, Zhou Y and Du Z: Tumor-suppressive function of long noncoding RNA MALAT1 in glioma cells by downregulation of MMP2 and inactivation of ERK/MAPK signaling. Cell Death Dis 7: e2123, 2016.

31. Cao S, Wang Y, Li J, Lv M, Niu H and Tian Y: Tumor-suppressive function of long noncoding RNA MALAT1 in glioma cells by suppressing miR-155 expression and activating FBXW7 function. Am J Cancer Res 6: 2561-2574, 2016.

32. Wang J, Ma S, Ma R, Qu X, Liu W, Lv C, Zhao S and Gong Y: KIF2A silencing inhibits the proliferation and migration of breast cancer cells and correlates with unfavorable prognosis in breast cancer. BMC Cancer 14: 461, 2014.

33. Iltzsche F, Simon K, Stopp S, Pattschull G, Francke S, Wolter P, Hauser S, Murphy DJ, Garcia P, Rosenwald A and Gaubatz S: An important role for Myb-MuvB and its target gene KIF23 in a mouse model of lung adenocarcinoma. Oncogene 36: 110-121, 2017.

34. Venere M, Horbinski C, Crish JF, Jin X, Vasanji A, Major J, Burrows AC, Chang C, Prokop J, Wu Q, et al: The mitotic kinesin KIF11 is a driver of invasion, proliferation, and self-renewal in glioblastoma. Sci Transl Med 7: 143r-304r, 2015.

35. Matsuda M, Yamamoto T, Matsumura A and Kaneda Y: Highly efficient eradication of intracranial glioblastoma using Eg5 siRNA combined with HVJ envelope. Gene Ther 16: 1465-1476, 2009.

36. Exertier P, Javerzat S, Wang B, Franco M, Herbert J, Platonova N, Winandy M, Pujol N, Nivelles O and Ormenese S: Impaired angiogenesis and tumor development by inhibition of the mitotic kinesin Eg5. Oncotarget 4: 2302-2316, 2013.

37. Chen S, Han M, Chen W, He Y, Huang B, Zhao P, Huang Q, Gao L, Qu X and Li X: KIF1B promotes glioma migration and invasion via cell surface localization of MT1-MMP. Oncol Rep 35: 971-977, 2015.

38. Liu M, Nadar VC, Kozielski F, Kozlowska M, Yu W and Baas PW: Kinesin-12, a mitotic microtubule-associated motor protein, impacts axonal growth, navigation and branching. J Neurosci 30: 14896-14906, 2010.

39. Xu M, Liu D, Dong Z, Wang X, Wang X, Liu Y, Baas PW and Liu M: Kinesin-12 influences axonal growth during zebrafish neural development. Cytoskeleton (Hoboken) 71: 555-563, 2015.

40. Scanlan MJ, Gout I, Gordon CM, Williamson B, Stockert E, Gure AO, Jäger D, Chen YT, Mackay A, O'Hare MJ and Old LJ: Humoral immunity to human breast cancer: Antigen definition and quantitative analysis of mRNA expression. Cancer Immun 1: $4,2001$.

41. Zou JX, Duan Z, Wang J, Sokolov A, Xu J, Chen CZ, Li JJ and Chen HW: Kinesin family deregulation coordinated by bromodomain protein ANCCA and histone methyltransferase MLL for breast cancer cell growth, survival, and tamoxifen resistance. Mol Cancer Res 12: 539-549, 2014.

42. Bidkhori G, Narimani Z, Hosseini Ashtiani S, Moeini A, Nowzari-Dalini A and Masoudi-Nejad A: Reconstruction of an integrated genome-scale co-expression network reveals key modules involved in lung adenocarcinoma. PLoS One 8: e67552, 2013.

This work is licensed under a Creative Commons

Attribution-NonCommercial-NoDerivatives 4.0 International (CC BY-NC-ND 4.0) License. 